\title{
Predictors of New-onset Diabetes After Kidney Transplantation During 2019-nCoV Pandemic: A Unison of Frequentist Inference and Narrow Al
}

\author{
Ahmed Al-Imam ${ }^{1,2,3 *}$ (D) Ali Abdulrahman Al-Tabbakh ${ }^{4}$ \\ ${ }^{1}$ Department of Anatomy and Cellular Biology, College of Medicine, University of Baghdad, Baghdad, Iraq; ${ }^{2}$ Department of \\ Computer Science and Statistics, PUMS Doctoral School, Poznan University of Medical Sciences, Poznan, Poland; ${ }^{3}$ Barts and \\ The London School of Medicine and Dentistry, Queen Mary University of London, London, United Kingdom; ${ }^{4}$ Department of \\ Nephrology, Erbil Teaching Hospital, Erbil, Kurdistan Region, Iraq
}

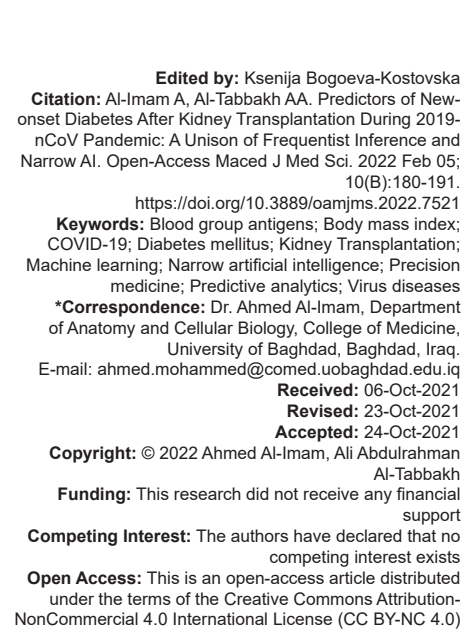

\section{Introduction}

Kidney transplantation is the best-known procedure for managing end-stage renal disease [1]. Despite so, new-onset diabetes after kidney transplant (NODAT) is a common and severe complication that occurs in $10-53 \%$ of transplant recipients who are not diagnosed as having diabetes before the transplantation [1], [2]. NODAT is also associated with an increased risk of renal allograft rejection, development of infections, and cardiovascular morbidities [3], [4]. Nonetheless, it was not until 2003 that the World Health Organization, following the American Diabetes Association (ADA), established the first international consensus guidelines for diagnosing it [5], [6]. Thus, NODAT refers strictly to patients not diagnosed with pre-transplant diabetes mellitus (DM) and acute infections, nor on a stable maintenance immunosuppressive regimen [7]. It is critical for epidemiological and clinical intentions to differentiate NODAT from other forms of post-transplant hyperglycemia, such as stress-induced hyperglycemia or transient post-transplant hyperglycemia [7].

The International Congress Guidelines stated that diagnosis of NODAT should fulfill either of the following conditions: (a) Fasting blood glucose $\geq 126 \mathrm{mg} / \mathrm{dL}$ on more than 1 occasion, (b) random blood glucose $\geq 200 \mathrm{mg} / \mathrm{dL}$ with symptoms, (c) postprandial blood glucose, $75 \mathrm{~g}$ oral glucose tolerance test $(2 \mathrm{~h}) \geq 200 \mathrm{mg} / \mathrm{dL}$, and (d) glycated hemoglobin $(\mathrm{HbA} 1 \mathrm{c}) \geq 6.5 \%$; according to the ADA, if a patient has discordant results from two different tests, then the test result above the diagnostic cutoff point should be repeated considering the possibility of $\mathrm{HbA} 1 \mathrm{c}$ assay interference [7]. We chose to implement the $\mathrm{HbA1c}$ percentage due to data availability. 
Risk factors related to NODAT can generally be categorized into modifiable and non-modifiable factors [8]. Modifiable factors include high body mass index (BMI), immunosuppressive therapeutics, including corticosteroids, and tacrolimus or cyclosporinecontaining regimens [8], [9]. Non-modifiable risk factors are associated with the inherent characteristics of the recipient, such as age, family history of DM, ethnicity, the presence of other diseases, such as hepatitis $C$ virus (HCV) and cytomegalovirus (CMV) [9], [10], [11].

Glucocorticoid-associated hyperglycemia often coincides with obesity and is usually due to acquired insulin resistance [12]. Several mechanisms can eventuate in glucocorticoid-induced insulin resistance; they exert their impact on metabolism via several tissues. For instance, they modulate lipid metabolism, leading to elevated levels of free fatty acids and an increase in insulin resistance; in addition, a suppressed pancreatic insulin secretion and $\beta$-cells apoptosis may lead to insulin resistance and glucocorticoid-associated hyperglycemia [13]. Diabetes develops some weeks or months after initiating oral glucocorticoids therapy; cyclosporine and tacrolimus also possess diabetogenic properties; calcineurin inhibitors (CNIs) can induce glucose intolerance by different mechanisms, including a decrease in insulin secretion, an increase in insulin resistance, and toxicity on the pancreatic $\beta$-cells [14], [15]. The effects of tacrolimus are more profound than cyclosporine; tacrolimus-specific binding protein (FKBP-12) is located in $\beta$-cells, which can potentiate the glucolipotoxicity, possibly by sharing common pathways of $\beta$-cell dysfunction; this is in contrast to the binding protein for cyclosporine (cyclophilin), which is preferentially located in the heart, liver, and kidney [16], [17].

DM has been cited among the most typical causes of chronic diseases of the kidney. More than $30 \%$ of non-diabetic transplant recipients experience new-onset diabetes after transplantation also referred to as post-transplant DM (PTDM) [18], [19]. According to Woodward et al. (2003), the incremental incidence of diabetes occurs mainly during the first 6 months post-transplantation and in individuals receiving high doses of immunosuppressive medication; further, the incidence of NODAT was 6 times higher among recipients during the 1 year of transplantation [20]. Palepu et al. (2015) examined the non-modifiable risk factors, including age which is considered the most decisive risk factor for evolving NODAT [21]. Cosio et al. (2001) studied 2078 allograft recipients; they confirmed that individuals over 45 years were almost 3 times more prone to develop post-transplant diabetes [22]. Concerning the modifiable risk factors, obesity was associated with the development of PTDM on many occasions; the analysis of the United States renal data system database revealed a significant effect of obesity $(p<0.0001)$ with a relative risk $(R R)$ of 1.73 [23]. In 2006, Shah et al. found that the risk of PTDM increased as the BMI increased; obese patients with a $\mathrm{BMI} \geq 30$ exhibited an RR value of $1.64(p<0.001)$ [24].

The current study aims to verify NODAT, its associated risk factors, and predictors in recipients within 1 year after kidney transplantation from a single center in Erbil City. Potential risk factors were not limited to weight, BMI, viral infections, and the effect of immunosuppressant therapy. The primary objective is to compare cases versus non-cases of NODAT and explore the predictors that influence the development of this entity in transplant recipients. The present study represents the first research from Iraq to explore NODAT predictors among kidney transplant recipients using frequentist statistics and artificial intelligence models.

\section{Methods}

\section{Ethical approval}

The study was conducted following the standard protocol of the ethical and scientific committee of Erbil Teaching Hospital (Erbil, Kurdistan). The authors abided by the Declaration of Helsinki by the World Medical Association, the European Union protocol for scientific purposes (EU Directive 210/63/EU), and the Framingham consensus of 1997 . We retrieved informed consent from each participant in the current study.

\section{Study design and participants}

The study is retrospective in design that aims to compare cases versus non-cases of DM following renal transplant and explore potential risk factors and predictors that influence the development of diabetes in transplant recipients. The total sample included 90 individuals $(n=90)$ of the Kurdish and Arabic ethnicities.

\section{Level of evidence}

The current research is a longitudinal observational study of a retrospective design. According to the Oxford Centre for Evidence-based Medicine (OCEBM), our study belongs to level-3 within the pyramidal hierarchy of the level of evidence.

\section{Diagnostic criteria and study variables}

We adopted the criteria for diagnosing DM of the ADA to categorize the transplant recipients into cases and non-cases of NODAT [7]. We collected several variables for each individual (Table 1), including age, gender, type of donor, blood group, primary kidney disease, the status of hemodialysis, BMI, $\mathrm{HbA} 1 \mathrm{c}$ levels, and the virology status concerning hepatitis $B$ virus 
Table 1: Study variables

\begin{tabular}{lll}
\hline Variable & Time & Abbreviation \\
\hline Age & $\mathrm{N} / \mathrm{A}$ & Age \\
Gender & $\mathrm{N} / \mathrm{A}$ & Gender \\
Type of donor & $\mathrm{N} / \mathrm{A}$ & Type of donor \\
Blood group & $\mathrm{N} / \mathrm{A}$ & Bl. Group \\
Blood Group (O+ve vs. others) & $\mathrm{N} / \mathrm{A}$ & Bl. Group (reduc.) \\
Primary kidney disease & $\mathrm{N} / \mathrm{A}$ & Prim. Kid. Dis. \\
Hemodialysis & $\mathrm{N} / \mathrm{A}$ & Hemodialysis \\
BMI & Transplant time, 3 months later & BMI (Peri-Tx), BMI (Post-Tx) \\
HbAC1 & Before the transplant, 6 months later & HbA1c (Pre-Tx), HbA1c (Post-Tx) \\
Hepatitis B virus & Before transplant & HBV \\
Hepatitis C virus & Before transplant & HCV \\
Cytomegalovirus & Before transplant & CMV \\
Induction with ATG (and/or) Simulect & Transplant time & Induction (ATG and Simulect) \\
Prednisolone & Transplant time, 3 months later & Pred. (Peri-Tx), Pred. (Post-Tx) \\
CsA & Transplant time, 3 months later & CsA (Peri-Tx), CsA (Post-Tx) \\
Post-transplant diabetes & C months after transplant & PTDM/NODAT \\
\hline${ }^{*}$ N/A: Not applicable, ${ }^{\star *}$ ATG: Anti-thymocyte globulin, Simulect: Basiliximab, HbA1c: Glycated hemoglobin, BMl: Body mass index, CsA: Cyclosporine A.
\end{tabular}

(HBV), HCV, and CMV. We also retrieved additional variables concerning managing the recipients with prednisolone, cyclosporine A (CsA), and induction therapy with immunosuppressive agents, including antithymocyte globulin (ATG) and basiliximab (Simulect).

\section{Statistical analysis and machine learning (ML)}

Data models and statistical analyses, including non-Bayesian statistics and ML, were conducted using IBM-SPSS version 24 and Microsoft Office Excel 2016 with the Analysis ToolPak plugin. The cutoff margin for statistical significance was at an alpha $(\alpha)$ value of 0.05 , equivalent to a $95 \%$ confidence interval $(95 \% \mathrm{Cl})$. We ran a series of parametric statistics, including independent t-test, Pearson's correlation, Chi-square test of independence, Fisher's exact test, and paired t-test. Eventually, we ran two summative ML models, which represent a modality of narrow artificial intelligence (nAl), by implementing multiple linear regression and neural network analysis; each has a predictors' importance analysis. Multiple linear regression deployed a forward stepwise regression. The neural network analysis utilized a multilayer perceptron neural network based on a scaled conjugate gradient optimization algorithm and a default SPSS allocation of the training set and testing set at $70-30 \%$ of the whole dataset.

\section{Results}

\section{participants}

\section{Demographic characteristics of the}

The total sample included 90 individuals $(n=90)$ of the Kurdish and Arabic ethnicities, distributed into males $(27.30 \%)$ and females $(63.70 \%)$, with a maleto-female ratio of $0.42-1$. Diabetic patients accounted for approximately one-third of the total sample ( $\mathrm{n} 1=32,35.6 \%$ ), while non-diabetics represented the rest $(\mathrm{n} 2=58,64.4 \%)$, at a ratio (cases: non-cases) of
0.55-1. Patients below the age of 18 years and those with diabetes before transplantation were excluded from the study.

\section{Description of the study sample}

Participants included males $(27.30 \%)$ and females $(63.70 \%)$, with live related $(16,17.8 \%)$ and unrelated allografts $(74,82.2 \%)$, with $(71,85.6 \%)$ and without prior management with hemodialysis $(13$, $14.4 \%)$. Most $(89,98.9 \%)$ had an induction with ATG, while only one individual received induction with both ATG and basiliximab (Simulect). Recipients were allocated into three main categories of primary kidney disease, including chronic kidney disease (CKD) (50, $55.6 \%)$, hypertension $(23,25.6 \%)$, and others $(17$, $18.9 \%)$. According to virology screening, almost onefifth of the sample $(19,21.1 \%)$ had CMV, while one-tenth were HCV positive $(9.10 \%)$, and only two individuals (2, $2.2 \%$ ) had HBV. Further, we noticed some association between blood groups $\mathrm{O}+\mathrm{ve}$ and developing NODAT; almost one-third $(27,30 \%)$ were O+ve, while the rest allocated into the remaining blood groups, including A- $(2,2.2 \%), A+(24,26.7 \%), A B-(2,2.2 \%), A B+(5$, $5.6 \%),(3,3.3 \%), B+(26,28.9 \%)$, and O- $(1,1.1 \%)$. Unfortunately, almost one-third of transplant recipients (32, 35.6\%) developed DM post-transplant. We also calculated descriptive statistics (the mean \pm the standard error of the mean) for all variables, including age (39.88 \pm 1.54$)$, BMI (Peri-Tx) $(23.86 \pm 0.55)$, BMI (Post-Tx) (25.54 \pm 0.49$), \mathrm{HbA} 1 \mathrm{c}$ (Pre-Tx) (5.12 \pm 0.04$)$, HbA1C (Post-Tx) (5.75 \pm 0.09$)$, CsA (Peri-Tx) (503.55 \pm 10.68), CsA (Post-Tx) (274.41 \pm 5.14 ), Pred. (Peri-Tx) (44.33 \pm 0.95$)$, and Pred. (Post-Tx) (9.67 \pm 0.13$)$.

We calculated the same descriptive parameters while stratifying the sample based on NODAT. Concerning age, those who had diabetes were older $(44.69 \pm 2.57$ vs. $37.22 \pm 1.86)$; those who became diabetic also had higher BMI, including BMI (Peri-Tx) $(25.70 \pm 0.99$ vs. $22.84 \pm 0.63)$ and BMI (Post-Tx) $(27.48 \pm$ 0.82 vs. $24.47 \pm 0.56)$. Diabetic patients also possessed higher levels of $\mathrm{HbA} 1 \mathrm{c}$, including $\mathrm{HbA} 1 \mathrm{c}$ (Pre-Tx) $(5.28$ \pm 0.07 vs. $5.04 \pm 0.04)$ and $\mathrm{HbA1C}$ (Post-Tx) $(6.70 \pm$ 0.09 vs. $5.22 \pm 0.05$ ). Further, individuals who developed 
Table 2: Descriptive statistics: Stratification by blood group

\begin{tabular}{|c|c|c|c|c|c|}
\hline \multirow[t]{2}{*}{ Blood group (reduced) } & \multirow{2}{*}{$\begin{array}{l}\mathrm{n} \\
\text { Statistic }\end{array}$} & \multirow{2}{*}{$\begin{array}{l}\text { Minimum } \\
\text { Statistic }\end{array}$} & \multirow{2}{*}{$\begin{array}{l}\text { Maximum } \\
\text { Statistic }\end{array}$} & \multicolumn{2}{|l|}{ Mean } \\
\hline & & & & Statistic & Std. error \\
\hline \multicolumn{6}{|l|}{$\overline{\mathrm{O}+}$} \\
\hline Age & 27 & 18 & 70 & 38.37 & 2.497 \\
\hline BMI (Peri-Tx) & 27 & 16.00 & 44.20 & 24.5667 & 1.14734 \\
\hline BMI (Post-Tx) & 27 & 18.70 & 40.00 & 26.3148 & 0.95091 \\
\hline HbA1c (Pre-Tx) & 27 & 3.90 & 5.90 & 5.0889 & 0.08083 \\
\hline HbA1c (Post-Tx) & 27 & 4.80 & 8.00 & 5.9815 & 0.16650 \\
\hline CsA (Peri-Tx) & 27 & 312.00 & 696.00 & 497.9259 & 18.35686 \\
\hline CsA (Post-Tx) & 27 & 200.00 & 420.00 & 279.6148 & 10.28227 \\
\hline Pred. (Peri-Tx) & 27 & 30 & 60 & 43.33 & 1.830 \\
\hline Pred. (Post-Tx) & 27 & 5 & 10 & 9.44 & 0.308 \\
\hline Valid N (listwise) & 27 & & & & \\
\hline \multicolumn{6}{|l|}{ Other } \\
\hline Age & 63 & 18 & 69 & 40.52 & 1.936 \\
\hline BMI (Peri-Tx) & 63 & 14.10 & 37.80 & 23.5508 & 0.62087 \\
\hline BMI (Post-Tx) & 63 & 17.10 & 39.10 & 25.2127 & 0.56073 \\
\hline HbA1c (Pre-Tx) & 63 & 4.20 & 6.00 & 5.1338 & 0.04300 \\
\hline HbA1c (Post-Tx) & 63 & 4.40 & 7.50 & 5.6444 & 0.10215 \\
\hline CsA (Peri-Tx) & 63 & 296.00 & 800.00 & 505.9587 & 13.16028 \\
\hline CsA (Post-Tx) & 63 & 184.00 & 396.80 & 272.1841 & 5.90795 \\
\hline Pred. (Peri-Tx) & 63 & 30 & 60 & 44.76 & 1.111 \\
\hline Pred. (Post-Tx) & 63 & 5 & 10 & 9.76 & 0.135 \\
\hline Valid N (listwise) & 63 & & & & \\
\hline
\end{tabular}

PTDM received higher doses of immunosuppressives, including CsA (Peri-Tx) (529.63 \pm 19.18 vs. $489.16 \pm$ 12.49), CsA (Post-Tx) (296.43 \pm 9.07 vs. $262.27 \pm 5.66)$, Pred. (Peri-Tx) (46.41 \pm 1.65 vs. $43.19 \pm 1.14)$, and Pred. (Post-Tx) $(9.53 \pm 0.26$ vs. $9.74 \pm 0.147)$. We also stratified the sample based on blood grouping (Table 2) and the primary kidney disease (Table 3).

Table 3: Descriptive statistics: Stratification by primary kidney disease

\begin{tabular}{|c|c|c|c|c|c|}
\hline \multirow[t]{2}{*}{ Primary kidney disease } & \multirow{2}{*}{$\begin{array}{l}\mathrm{n} \\
\text { Statistic }\end{array}$} & \multirow{2}{*}{$\begin{array}{l}\text { Minimum } \\
\text { Statistic }\end{array}$} & \multirow{2}{*}{$\begin{array}{l}\text { Maximum } \\
\text { Statistic }\end{array}$} & \multicolumn{2}{|l|}{ Mean } \\
\hline & & & & Statistic & Std. error \\
\hline \multicolumn{6}{|l|}{ CKD } \\
\hline Age & 50 & 18 & 69 & 36.22 & 1.940 \\
\hline BMI (Peri-Tx) & 50 & 14.10 & 34.50 & 22.6900 & 0.61581 \\
\hline BMI (Post-Tx) & 50 & 17.10 & 35.00 & 24.5500 & 0.55781 \\
\hline HbA1c (Pre-Tx) & 50 & 3.90 & 5.90 & 5.0926 & 0.04941 \\
\hline HbA1c (Post-Tx) & 50 & 4.60 & 8.00 & 5.8060 & 0.12350 \\
\hline CsA $[$ Peri-Tx $]$ & 50 & 296.00 & 688.00 & 482.6080 & 12.84144 \\
\hline CsA (Post-Tx) & 50 & 184.00 & 361.60 & 264.8160 & 5.74136 \\
\hline Pred. [Peri-Tx] & 50 & 30 & 60 & 42.80 & 1.326 \\
\hline Pred. (Post-Tx) & 50 & 5 & 10 & 9.60 & 0.194 \\
\hline Valid N (listwise) & 50 & & & & \\
\hline \multicolumn{6}{|l|}{ HPT } \\
\hline Age & 23 & 37 & 70 & 52.61 & 1.938 \\
\hline BMI $[$ Peri-Tx] & 23 & 19.00 & 37.80 & 27.2652 & 0.99799 \\
\hline BMI (Post-Tx) & 23 & 20.70 & 39.10 & 28.1130 & 0.94788 \\
\hline HbA1c (Pre-Tx) & 23 & 4.40 & 6.00 & 5.1913 & 0.08480 \\
\hline HbA1c (Post-Tx) & 23 & 4.40 & 7.20 & 5.7435 & 0.17758 \\
\hline CsA (Peri-Tx) & 23 & 428.00 & 800.00 & 581.9565 & 19.90417 \\
\hline CsA (Post-Tx) & 23 & 244.00 & 396.80 & 306.3826 & 9.51851 \\
\hline Pred. (Peri-Tx) & 23 & 30 & 60 & 48.70 & 1.413 \\
\hline Pred. (Post-Tx) & 23 & 10 & 10 & 10.00 & 0.000 \\
\hline Valid N (listwise) & 23 & & & & \\
\hline \multicolumn{6}{|l|}{ Other } \\
\hline Age & 17 & 18 & 58 & 33.41 & 3.254 \\
\hline BMI (Peri-Tx) & 17 & 16.00 & 44.20 & 22.6706 & 1.55423 \\
\hline BMI (Post-Tx) & 17 & 18.30 & 40.00 & 24.9882 & 1.30392 \\
\hline HbA1c (Pre-Tx) & 17 & 4.30 & 5.90 & 5.1059 & 0.08722 \\
\hline HbA1c (Post-Tx) & 17 & 4.60 & 7.00 & 5.5706 & 0.17506 \\
\hline CsA (Peri-Tx) & 17 & 360.00 & 696.00 & 459.0588 & 20.36586 \\
\hline CsA (Post-Tx) & 17 & 200.00 & 420.00 & 259.3882 & 13.96458 \\
\hline Pred. (Peri-Tx) & 17 & 30 & 60 & 42.94 & 2.189 \\
\hline Pred. (Post-Tx) & 17 & 5 & 10 & 9.41 & 0.403 \\
\hline Valid N (listwise) & 17 & & & & \\
\hline
\end{tabular}

Diabetics versus non-diabetics, and blood groups' differential effect

Independent (unpaired) t-test validated a significant difference in favor of diabetic patients for all the variables except for medicating with prednisolone (Table 4). Significant differences existed for age, BMI,
$\mathrm{HbA1c}$ level, and medicating with cyclosporine. The biggest significant difference was for HbA1c (Post-Tx) $(t=15.428, p<0.001$, Cohen's $d=3.26)$, while the least significant difference was for CsA (Peri-Tx) $(t=1.837$, $p=0.070$, Cohen's $d=0.40)$; to summarize, the largest effect size was related to $\mathrm{HbA} 1 \mathrm{c}$ level post-transplant, while the weakest was for medicating with cyclosporine.

Concerning the association of blood grouping and developing DM following a renal transplant, independent t-testing (Table 5) yielded a significant difference in favor of recipients with O+ve blood group concerning one variable only, HbA1c (Post-Tx) (1.771, $p=0.080$, Cohen's $d=0.40)$; the difference concerning $\mathrm{HbA} 1 \mathrm{c}$ level post-transplant is conditionally significant at $90 \% \mathrm{Cl}$ and possessed a medium effect size.

\section{correlations \\ Association matrices: Pearson's}

Concerning the whole sample, Pearson's bivariate correlations confirmed significant associations between most of the variables. Most correlations were strongly significant, that is, at $99 \% \mathrm{Cl}$, and all correlations were positive. At the same time, there was only one inverse correlation between $\mathrm{HbA} 1 \mathrm{c}$ level and prednisolone dose post-transplant (Pearson's $r=-0.215, p=0.041)$. Further, most of the correlations had either a medium or a large effect size, the strongest of which existed for BMI versus CsA.

We also conducted the bivariate correlations while stratifying the sample into cases and non-cases of NODAT (Table 6). Generally speaking, there were more significant associations among the variables within non-diabetic individuals; those correlations were more abundant, of higher statistical significance, and had a larger effect size than diabetic patients. To summarize, fewer significant associations were present within diabetic patients, and none of which had an inverse correlation; most of the significant correlations had a medium effect size. Further, and in harmony with the correlations concerning the non-stratified sample, the strongest correlations existed between BMI and CsA.

\section{Exploration of categorical variables}

We explored the association among categorical variables using Pearson's Chi-square test (Chi-square test of independence) and Fisher's exact test. We could not detect any significant association between gender and $\mathrm{DM}$, the type of donor and DM, blood grouping and DM, the primary kidney disease and DM, hemodialysis and DM, HBV and DM, and the mode of induction and DM. Nonetheless, there was a significant association between HCV and DM ( $p=0.001$, Cramer's V =0.371), and between CMV and DM ( $p=0.080$, Cramer's $V=0.185)$ (Figure 1). 
Table 4: Diabetics versus non-diabetics: Independent t-test

\begin{tabular}{|c|c|c|c|c|c|}
\hline \multirow[t]{2}{*}{ Variable } & \multicolumn{2}{|c|}{ Levene's test for equality of variances } & \multicolumn{3}{|c|}{ t-test for equality of means } \\
\hline & $F$ & Sig. & $\mathrm{t}$ & Sig. (two tailed) & Mean difference \\
\hline \multicolumn{6}{|l|}{ Age } \\
\hline Equal variances assumed & 0.026 & 0.872 & 2.373 & 0.020 & 7.463 \\
\hline Equal variances not assumed & & & 2.355 & 0.022 & 7.463 \\
\hline \multicolumn{6}{|l|}{ BMI (Peri-Tx) } \\
\hline Equal variances assumed & 0.737 & 0.393 & 2.549 & 0.013 & 2.85722 \\
\hline Equal variances not assumed & & & 2.436 & 0.018 & 2.85722 \\
\hline \multicolumn{6}{|l|}{ BMI (Post-Tx) } \\
\hline Equal variances assumed & 0.086 & 0.771 & 3.108 & 0.003 & 3.00711 \\
\hline Equal variances not assumed & & & 3.032 & 0.004 & 3.00711 \\
\hline Equal variances assumed & 3.996 & 0.049 & 3.131 & 0.002 & 0.24000 \\
\hline Equal variances not assumed & & & 2.876 & 0.006 & 0.24000 \\
\hline \multicolumn{6}{|l|}{ HbA1c (Post-Tx) } \\
\hline Equal variances assumed & 1.069 & 0.304 & 15.428 & 0.000 & 1.48588 \\
\hline Equal variances not assumed & & & 14.289 & 0.000 & 1.48588 \\
\hline \multicolumn{6}{|l|}{ CsA (Peri-Tx) } \\
\hline Equal variances assumed & 0.204 & 0.652 & 1.837 & 0.070 & 40.46293 \\
\hline Equal variances not assumed & & & 1.768 & 0.082 & 40.46293 \\
\hline \multicolumn{6}{|l|}{ CsA (Post-Tx) } \\
\hline Equal variances assumed & 0.505 & 0.479 & 3.360 & 0.001 & 34.15603 \\
\hline \multicolumn{6}{|l|}{ Pred. (Peri-Tx) } \\
\hline Equal variances assumed & 0.022 & 0.883 & 1.638 & 0.105 & 3.217 \\
\hline Equal variances not assumed & & & 1.602 & 0.114 & 3.217 \\
\hline \multicolumn{6}{|l|}{ Pred. (Post-Tx) } \\
\hline Equal variances assumed & 2.294 & 0.133 & -0.759 & 0.450 & -0.210 \\
\hline Equal variances not assumed & & & -0.700 & 0.487 & -0.210 \\
\hline
\end{tabular}

BMI: Body mass index, HbA1c: Glycated hemoglobin, CsA: Cyclosporine A.

Table 5: Blood groups: Independent t-test

\begin{tabular}{|c|c|c|c|c|c|}
\hline \multirow[t]{2}{*}{ Variable } & \multicolumn{2}{|c|}{ Levene's test for equality of variances } & \multicolumn{3}{|c|}{ t-test for equality of means } \\
\hline & $\mathrm{F}$ & Sig. & $\mathrm{t}$ & Sig. (two tailed) & Mean difference \\
\hline \multicolumn{6}{|l|}{ Age } \\
\hline Equal variances assumed & 3.429 & 0.067 & -0.637 & 0.526 & -2.153 \\
\hline Equal variances not assumed & & & -0.682 & 0.498 & -2.153 \\
\hline \multicolumn{6}{|l|}{ BMI (Peri-Tx) } \\
\hline Equal variances assumed & 0.318 & 0.574 & 0.840 & 0.403 & 1.01587 \\
\hline Equal variances not assumed & & & 0.779 & 0.441 & 1.01587 \\
\hline \multicolumn{6}{|l|}{ BMI (Post-Tx) } \\
\hline Equal variances assumed & 0.187 & 0.667 & 1.041 & 0.301 & 1.10212 \\
\hline Equal variances not assumed & & & 0.998 & 0.323 & 1.10212 \\
\hline \multicolumn{6}{|l|}{ HbA1c (Pre-Tx) } \\
\hline Equal variances assumed & 0.480 & 0.490 & -0.533 & 0.595 & -0.04492 \\
\hline Equal variances not assumed & & & -0.491 & 0.626 & -0.04492 \\
\hline \multicolumn{6}{|l|}{ HbA1c (Post-Tx) } \\
\hline Equal variances assumed & 0.014 & 0.907 & 1.771 & 0.080 & 0.33704 \\
\hline Equal variances not assumed & & & 1.725 & 0.091 & 0.33704 \\
\hline \multicolumn{6}{|l|}{ CsA (Peri-Tx) } \\
\hline Equal variances assumed & 0.100 & 0.753 & -0.343 & 0.733 & -8.03280 \\
\hline Equal variances not assumed & & & -0.356 & 0.724 & -8.03280 \\
\hline \multicolumn{6}{|l|}{ CsA (Post-Tx) } \\
\hline Equal variances assumed & 0.182 & 0.671 & 0.660 & 0.511 & 7.43069 \\
\hline Equal variances not assumed & & & 0.627 & 0.534 & 7.43069 \\
\hline \multicolumn{6}{|l|}{ Pred. (Peri-Tx) } \\
\hline Equal variances assumed & 1.041 & 0.310 & -0.688 & 0.493 & -1.429 \\
\hline Equal variances not assumed & & & -0.667 & 0.508 & -1.429 \\
\hline \multicolumn{6}{|l|}{ Pred. (Post-Tx) } \\
\hline Equal variances assumed & 4.803 & 0.031 & -1.102 & 0.274 & -0.317 \\
\hline Equal variances not assumed & & & -0.943 & 0.352 & -0.317 \\
\hline
\end{tabular}

BMI: Body mass index, HbA1c: Glycated hemoglobin, CsA: Cyclosporine A.

Table 6: Correlation matrix: Pearson's correlation for diabetic patients

\begin{tabular}{|c|c|c|c|c|c|c|c|c|c|}
\hline Variable & Age & BMI (Peri-Tx) & BMI (Post-Tx) & HbA1c (Pre-Tx) & HbA1c (Post-Tx) & CsA (Peri-Tx) & CsA (Post-Tx) & Pred. (Peri-Tx) & Pred. (Post-Tx) \\
\hline \multicolumn{10}{|l|}{ Age } \\
\hline Pearson's r & 1 & 0.296 & 0.238 & 0.107 & 0.095 & $0.429^{*}$ & $0.369^{*}$ & 0.133 & -0.225 \\
\hline $\mathrm{p}$-value & & 0.099 & 0.190 & 0.561 & 0.606 & 0.014 & 0.038 & 0.469 & 0.216 \\
\hline \multicolumn{10}{|l|}{ BMI (Peri-Tx) } \\
\hline Pearson's $r$ & 0.296 & 1 & $0.918^{\star *}$ & $0.356^{*}$ & 0.056 & $0.729^{\star \star}$ & $0.787^{\star *}$ & 0.236 & 0.072 \\
\hline $\mathrm{p}$-value & 0.099 & & 0.000 & 0.046 & 0.760 & 0.000 & 0.000 & 0.193 & 0.696 \\
\hline \multicolumn{10}{|l|}{ BMI (Post-Tx) } \\
\hline Pearson's $r$ & 0.238 & $0.918^{* *}$ & 1 & 0.290 & 0.200 & $0.689^{* *}$ & $0.840^{* *}$ & 0.196 & 0.010 \\
\hline $\mathrm{p}$-value & 0.190 & 0.000 & & 0.108 & 0.271 & 0.000 & 0.000 & 0.282 & 0.955 \\
\hline \multicolumn{10}{|l|}{ HbA1c (Pre-Tx) } \\
\hline Pearson's r & 0.107 & $0.356^{*}$ & 0.290 & 1 & 0.055 & $0.356^{*}$ & 0.274 & 0.039 & 0.112 \\
\hline $\mathrm{p}$-value & 0.561 & 0.046 & 0.108 & & 0.764 & 0.045 & 0.129 & 0.834 & 0.542 \\
\hline \multicolumn{10}{|c|}{ HbA1c (Post-Tx) } \\
\hline Pearson's r & 0.095 & 0.056 & 0.200 & 0.055 & 1 & 0.225 & 0.249 & 0.127 & -0.339 \\
\hline $\mathrm{p}$-value & 0.606 & 0.760 & 0.271 & 0.764 & & 0.217 & 0.170 & 0.488 & 0.058 \\
\hline \multicolumn{10}{|l|}{ CsA (Peri-Tx) } \\
\hline Pearson's $r$ & $0.429^{*}$ & $0.729^{\star *}$ & $0.689^{* *}$ & $0.356^{*}$ & 0.225 & 1 & $0.867^{\star \star}$ & $0.461^{* *}$ & -0.116 \\
\hline $\mathrm{p}$-value & 0.014 & 0.000 & 0.000 & 0.045 & 0.217 & & 0.000 & 0.008 & 0.529 \\
\hline \multicolumn{10}{|l|}{ CsA (Post-Tx) } \\
\hline Pearson's $r$ & $0.369^{*}$ & $0.787^{\star \star}$ & $0.840^{\star *}$ & 0.274 & 0.249 & $0.867^{\star *}$ & 1 & $0.453^{* *}$ & -0.130 \\
\hline$p$-value & 0.038 & 0.000 & 0.000 & 0.129 & 0.170 & 0.000 & & 0.009 & 0.479 \\
\hline \multicolumn{10}{|l|}{ Pred. (Peri-Tx) } \\
\hline Pearson's r & 0.133 & 0.236 & 0.196 & 0.039 & 0.127 & $0.461^{* *}$ & $0.453^{\star *}$ & 1 & -0.126 \\
\hline $\mathrm{p}$-value & 0.469 & 0.193 & 0.282 & 0.834 & 0.488 & 0.008 & 0.009 & & 0.494 \\
\hline \multicolumn{10}{|l|}{ Pred. (Post-Tx) } \\
\hline Pearson's $r$ & -0.225 & 0.072 & 0.010 & 0.112 & -0.339 & -0.116 & -0.130 & -0.126 & 1 \\
\hline p-value & 0.216 & 0.696 & 0.955 & 0.542 & 0.058 & 0.529 & 0.479 & 0.494 & \\
\hline
\end{tabular}




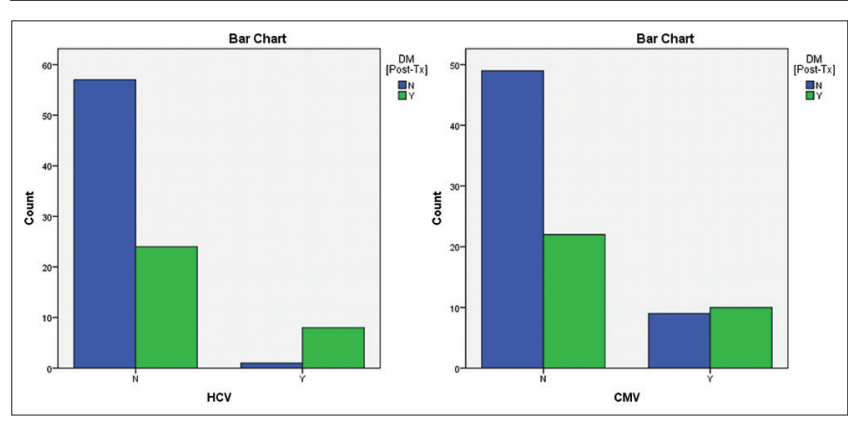

Figure 1: Association of diabetes mellitus with hepatitis $C$ virus and cytomegalovirus

To summarize, there was no significant association among NODAT and the categorical variables, except for HCV and CMV. The association between HCV and NODAT was strongly significant (at $99.9 \% \mathrm{Cl}$ ) and had a larger effect size than the association between CMV and NODAT, which is conditionally significant at a lower $\alpha$ value $(90 \%$ $\mathrm{Cl})$.

To further validate the results from the Chi-square test of independence concerning the association of virology +ve patients (HCV and CMV) with DM, we conducted a collateral independent t-test by comparing the levels of $\mathrm{HbA} 1 \mathrm{c}$ among those who tested either positive or negative for each of HCV and CMV. Unpaired t-testing verified a significant difference in favor of individuals who are HCV +ve $(t=4.831, p<0.001$, Cohen's $d=$ 1.66); similarly, a significant difference existed in favor of CMV +ve transplant recipients concerning their HbA1c levels $(t=2.453, p=0.016$, Cohen's $d=0.66)$. In conclusion, the Chi-square test of independence and t-testing was in unison; both detected a significant association of NODAT with each of HCV and CMV.

\section{$B M I$ and HbA1c: Pre- and post-transplant}

We also deployed paired (dependent) t-test concerning two variables, $\mathrm{BMI}$ and $\mathrm{HbA} 1 \mathrm{c}$; there was a significant difference between BMI (Peri-Tx) versus BMI (Post-Tx) $(t=-6.815, p<0.001$, Cohen's $d=0.34)$ and HbA1c (Pre-Tx) versus HbA1c (Post-Tx) $(t=-7.565$, $p<0.001$, Cohen's $d=0.97)$. Hence, each variable changed significantly following renal transplant; both were significantly different at $99.9 \% \mathrm{Cl}$, while $\mathrm{HbA} 1 \mathrm{c}$ possessed a larger effect size than the BMI.

\section{Narrow Al: Supervised ML}

Finally, we implemented two ML models; the first deployed multiple linear regression and predictors' importance analysis, using automatic linear modeling in SPSS. Linear modeling was guided by the cumulative results from earlier non-Bayesian statistical testing and causality reasoning based on the renowned Bradford Hill criteria [18]. regression

\section{Linear modeling: Multiple linear}

Multiple linear regression deployed a forward stepwise regression while feeding the model with $\mathrm{HbA} 1 \mathrm{c}$ as the dependent (outcome) variable and the other variables as the independent (predictor) variables, including HCV, HbA1c (Pre-Tx), BMI, CMV, hemodialysis, and blood group. The holistic model detected a significant effect of the predictors on $\mathrm{HbA} 1 \mathrm{c}$ levels $\left(F=10.637, p<0.001\right.$, adjusted $R^{2}=$ 0.464 , model accuracy $=46.4 \%$ ) (Figure 2$)$; the value of the coefficient of determination $\left(R^{2}\right)$ entails the presence of covert (unknown) predictors that can explain the full variance within the outcome. Further, according to our model, predictors' importance analysis verified a significant effect of six variables at $95 \% \mathrm{Cl}$, including $\mathrm{HCV}$ (predictor's importance $=0.247, p<0.001), \mathrm{HbA} 1 \mathrm{c}($ Pre-Tx $)$ (0.154, $p=0.005)$, BMI (Post-Tx) $(0.152, p=0.005)$, CMV (0.151, $p=0.006)$, hemodialysis $(0.099, p=$ $0.023)$, and blood group $(0.077, p=0.046)$. The model also detected a significant effect of two variables at $90 \% \mathrm{Cl}$, including Pred. (Post-Tx) $(0.060, p=0.77)$ and BMI (Peri-Tx) $(0.059, p=$ 0.079) (Figure 3).

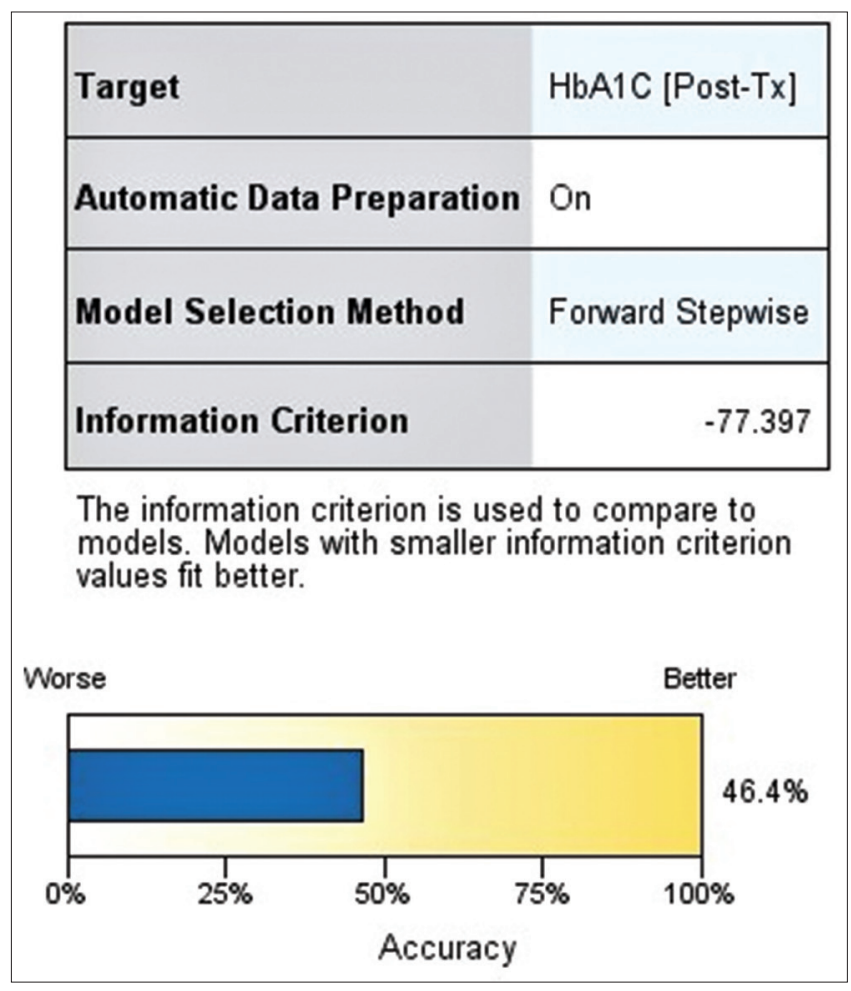

Figure 2: Multiple linear regression: Model summary perceptron

\section{Artificial neural network (ANN): Multilayer}

The second ML model relied on an ANN analysis, in which we fed the model with several potential predictors (Table 7) that may affect the outcome (PTDM). We 


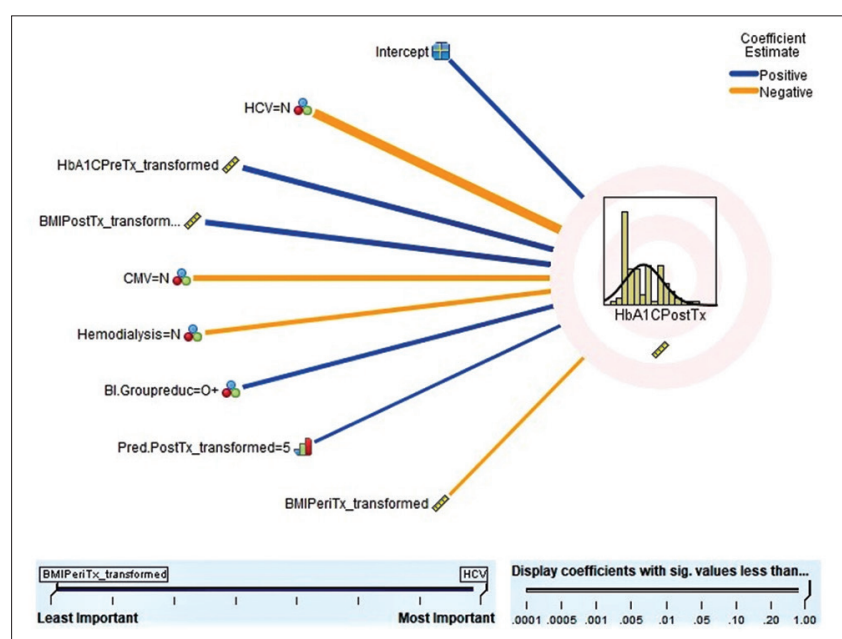

Figure 3: Multiple linear regression: Predictors' importance analysis

selected the independent variables marked as significant in the previous frequentist models and multiple linear regression. The ANN was fully accurate $(100 \%)$ in predicting cases and non-cases of diabetes; it has a perfect receiver operating characteristic curve, with an area under the curve (area under curve =1) for cases and non-cases of DM (Figure 4). Further, the independent variables' importance analysis assigned the highest weight (importance), in descending order, to BMI (Post-Tx) (predictor's importance $=0.205$, normalized importance $=100.00 \%)$, BMI $($ Peri-Tx) $(0.2$, $97.30 \%)$, age $(0.182,88.60 \%), \mathrm{HbA} 1 \mathrm{c}$ (Pre-Tx) $(0.171$, $83.40 \%), \operatorname{HCV}(0.126,61.50 \%), \operatorname{CMV}(0.079,38.60 \%)$, and blood group (reduced) $(0.036,17.30 \%)$ (Figure 5).

Table 7: ANN analysis: Network information summary

\begin{tabular}{|c|c|c|}
\hline Input layer & & \\
\hline \multirow[t]{7}{*}{ Factors } & 1 & Age \\
\hline & 2 & Blood group [reduced] \\
\hline & 3 & BMI (Peri-Tx) \\
\hline & 4 & BMI (Post-Tx) \\
\hline & 5 & HbA1c (Pre-Tx) \\
\hline & 6 & $\mathrm{HCV}$ \\
\hline & 7 & CMV \\
\hline Number of units ${ }^{a}$ & & 170 \\
\hline \multicolumn{3}{|l|}{ Hidden layer (s) } \\
\hline Number of hidden layers & & 1 \\
\hline Number of units in hidden layer $1^{\mathrm{a}}$ & & 9 \\
\hline Activation function & & Hyperbolic tangent \\
\hline \multicolumn{3}{|l|}{ Output layer } \\
\hline Dependent variables & 1 & DM (Post-Tx) \\
\hline Number of units & & 2 \\
\hline Activation function & & Softmax \\
\hline Error function & & Cross-entropy \\
\hline
\end{tabular}

\section{Discussion}

\section{DM and chronic kidney diseases}

DM has been cited as one of the most frequent causes of CKD. On the other side, over $30 \%$ of nondiabetic transplant recipients experience NODAT, also referred to as PTDM [18], [19]. In the present study, 32 out of 90 recipients $(35.6 \%)$ developed NODAT after renal transplantation. The incremental incidence of diabetes occurs mainly during the first 6 months

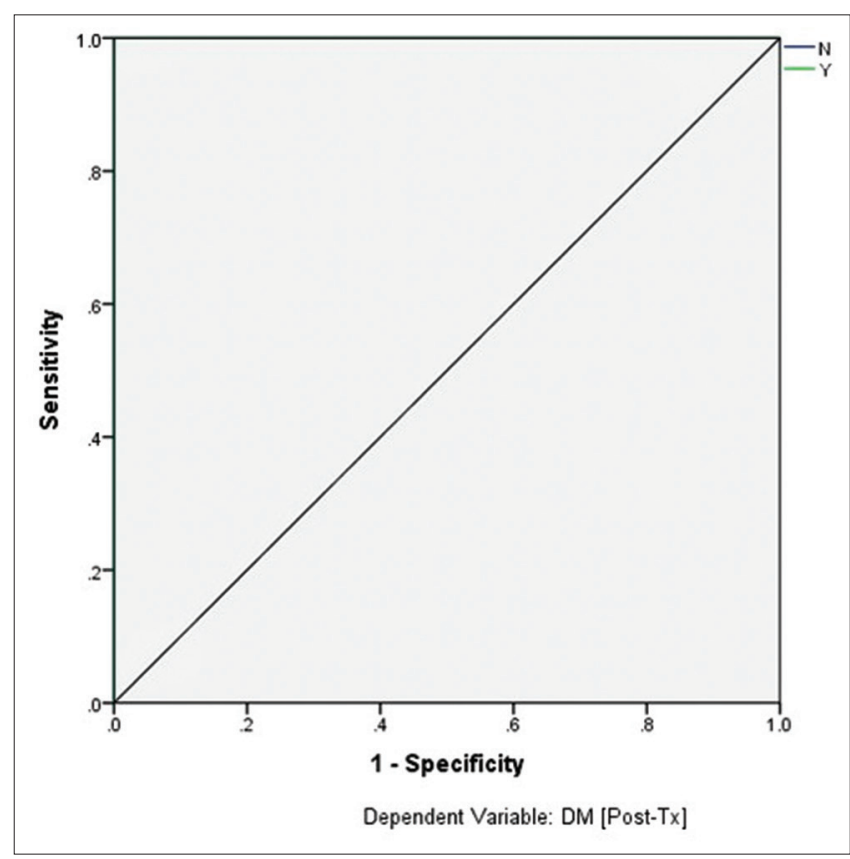

Figure 4: Neural network: Receiver operating characteristic curve

post-transplantation, in individuals receiving high doses of immunosuppressive medication; the incidence of NODAT is 6 times higher among recipients during the 1 year of transplantation [20].

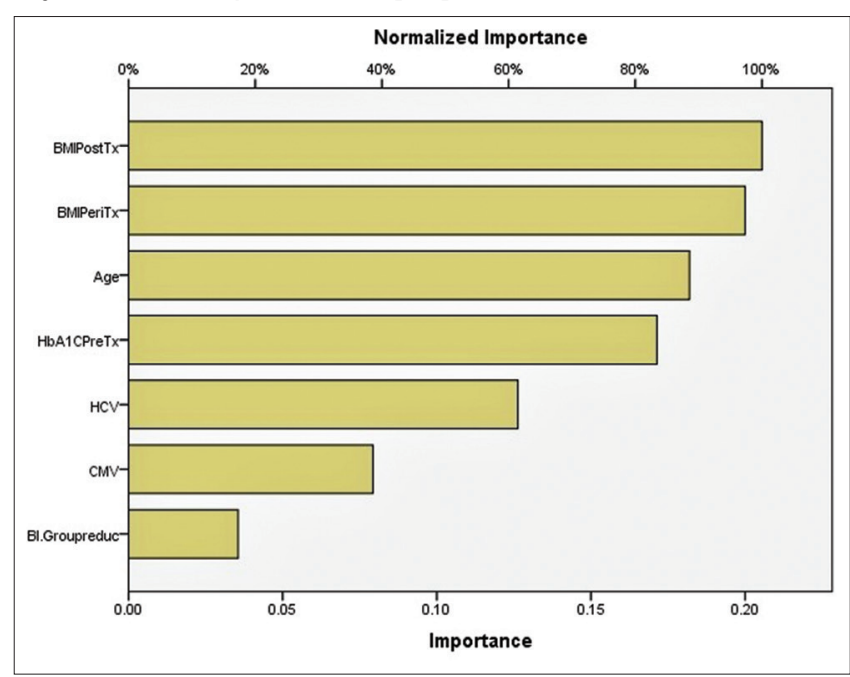

Figure 5: Neural network: Predictors' importance analysis

Immunosuppressive medications are potential diabetogenic agents; they possess several mechanisms to induce insulin resistance. For instance, steroids modulate lipid metabolism, leading to elevated levels of free fatty acids; they can also suppress pancreatic insulin secretion and induce $\beta$-cells apoptosis resulting in glucocorticoid-associated hyperglycemia [13]. CNIs can induce glucose intolerance by similar mechanisms; they can influence a decrease in insulin secretion, toxicity on the pancreatic $\beta$-cells, and culminate in insulin resistance status [14], [15]. The high risk of developing NODAT within the 1 month following the transplantation may relate to all the previously mentioned mechanisms attributed to immunosuppressives. Further, the immune 
system status and the genetic buildup of the transplant recipients, including their human leukocyte antigen (HLA) profile, can be pivotal in the rapid evolvement of post-transplant diabetes within half to 1 year from the transplant procedure; the process is most likely to be multifaceted due to elaborate interactions among several explanatory variables.

\section{Modifiable and non-modifiable risk factors of NODAT}

According to Palepu et al. (2015), among the non-modifiable risk factors, age is considered the most decisive risk factor for the development of PTDM [21]. Cosio et al. (2001) studied 2078 allograft recipients; they confirmed that individuals older than 45 years were 2.9 times more prone to develop PTDM than those who were younger at the time of transplantation [22]. As far as the modifiable risk factors are concerned, obesity was found in association with the development of PTDM in many cases; analysis of the United States Renal Data System database revealed that the RR of obesity amounts to $1.73(p<0.0001)$ [23]. Shah et al. (2006) found that the risk of PTDM increased as BMI increased; obese patients (BMI $\geq 30 \mathrm{~kg} / \mathrm{m}^{2}$ ) exhibited an RR value of $1.64(p<0.001)$ [24]. Similarly, in the current study, obesity was determined as a predictor of PTDM.

Obese and older individuals can have higher levels of free fatty acids, glycerol, hormones, reactive oxygen species, pro-inflammatory mediators, and inflammatory cytokines that can lead to insulin resistance. The diabetogenic process is principally related to the impairment of pancreatic $\beta$-islet cells' functions, causing a lack of control over blood glucose level; besides, the development of diabetes becomes inevitable if the $\beta$-islet cells' failure is accompanied by insulin resistance.

\section{CNIs and kidney transplants}

Several published reports showed a higher incidence of NODAT following the introduction of CNIs in renal transplantation [25]. Other prior studies showed no difference between the two $\mathrm{CNI}$ in developing NODAT [26]. We did not compare tacrolimus and cyclosporine; transplant recipients on tacrolimus were excluded from our research.

\section{Virology screening}

The previous studies suggested that asymptomatic CMV infection and CMV disease are independently associated with the development of NODAT, while other studies reported that CMV was not a risk factor [27], [28]. In the current study, half of the recipients with CMV infection developed NODAT, and that HCV infection was significantly $(p<0.001)$ associated with a higher incidence of diabetes. A meta-analysis confirmed a relationship between $\mathrm{HCV}$ infection and NODAT with an approximately 4 times greater risk of NODAT in HCV-infected recipients [29], [30]. In HCV-infected recipients, NODAT usually manifests in the 1 month after transplantation when higher doses of immunosuppressants are administered [31].

$\mathrm{CMV}$ and $\mathrm{HCV}$ can induce diabetes in transplant recipients by affecting the liver and pancreatic functions, that is, the hepatopancreatobiliary unit, and possibly by inducing peripheral insulin resistance. Besides, the therapeutic regimes for managing these conditions, including interferons, may also affect hepatopancreatic functions. Moreover, there could be a bidirectional interaction between the viral infection and the status of insulin resistance; it can lead to a vicious cycle augmenting each other. For instance, diabetic patients and their medications can lead to an immunocompromised status that causes a flare-up of the viral infection. At the same time, these viruses can potentiate the diabetogenic cycle even further.

\section{HbA1c and kidney transplant}

Assessment of the pre-transplant $\mathrm{HbA} 1 \mathrm{c}$ levels may be a valuable tool for an early diagnosis of NODAT in kidney transplant recipients. In the present study, NODAT patients showed higher pre-transplant $\mathrm{BMI}$ and $\mathrm{HbA} 1 \mathrm{c}$ than those without NODAT. Our results are in unison with Shin et al. (2017), in which they studied 1499 non-diabetic primary kidney transplant recipients and verified an association between higher pre-transplantation HbA1c level and PTDM; Shin et al. found that 395 recipients $(26.4 \%)$ developed PTDM over a median follow-up time of 1.8 years [32].

According to Tillmann and fellows (2018), renal transplant recipients were followed up for 4 years post-transplantation, and they were managed with cyclosporine and prednisolone-based regimen; they showed an increasing $\mathrm{HbA} 1 \mathrm{c}$ level and increased risk of developing pre-diabetes in recipients on low-dose prednisolone [33]. In contrast, our study confirmed no significant relationship between higher levels of $\mathrm{HbA} 1 \mathrm{c}$ and prednisolone therapy ( $\mathrm{p}=0.817$ ). Besides, Johannes et al. (2002) confirmed that glucose metabolism improved after corticosteroid $(10 \mathrm{mg}$ of prednisolone) withdrawal; further dosage reduction under $5 \mathrm{mg} /$ day did not convey a tangible improvement concerning glucose metabolism [34].

\section{The rationale for deploying $n A l$ and $M L$}

The composite of non-Bayesian and nAl models can yield superior results for inferential purposes [35], [36], [37]. The rationale for using ML involves several key reasons [38]; it provides (1) collateral evidence based on ML algorithms, (2) an alternative method to classical data analytics, 
(3) reconciliation of non-Bayesian statistical models, including the univariate and multivariate models, with nAl models, (4) a form of convergent thinking, dealing with the research question from an alternative perspective, (5) a novel problem-solving approach, and (6) an innovative research method that can serve as a blueprint for future research within the discipline of transplant medicine, nephrology, and endocrinology.

\section{pandemic}

Kidney transplant, NODAT, and the

During the era of the severe acute respiratory syndrome (SARS-CoV-2) pandemic, also known as coronavirus disease 2019 (COVID-19) caused by the novel coronavirus 2019 (2019-nCoV), there have been unprecedented restrictions and obstacles within and beyond the healthcare system and the economy, due to the status of anomie imposed by the pandemic [39], [40], [41]. As of September 28, 2021, the number of confirmed infections exceeded 233,158,402 worldwide and 1,996,214 in Iraq; complications related to the illness claimed the lives of over 4,771,151 globally and 22,142 in Iraq; the pandemic affected nations from the developed and developing world, including the United States, India, Brazil, the United Kingdom, Russia, Turkey, France, Iran, Argentina, and Columbia [42].

We collected our data for transplant recipients during the pandemic itself. Nonetheless, there was no information on the infection status with SARS-CoV-2. The current study can guide subsequent research on the importance of including another novel potential predictor of NODAT, the SARS-CoV-2 virology screening. It is established that the COVID-19 is a systemic disease that affects multiple organs, including the kidneys [43]. Further, due to the vascular-based pathophysiology of the 2019-nCoV, kidney transplant procedures can be delayed, while the incidence of NODAT may also variate based on serostatus concerning SARS-CoV-2, as some kidney transplant recipients either had a 2019$\mathrm{nCoV}$ infection or received the COVID-19 vaccination; these are novel potential explanatory variables for the evolution of NODAT that mandate future investigations and experimentations [43], [44], [45], [46].

\section{Succeeding research}

\section{Precision transplant medicine}

Finally, the current study can be valuable for precision medicine, an emerging discipline of medical sciences that explore data from the genetic makeup of an individual, the environment, and the lifestyle to select the optimum treatment for that individual; it is a medicine that integrates information about a person's genes or proteins to prevent, diagnose, or manage a specific disease [47], [48]. According to Ashley (2016),
"Precision medicine describes the definition of disease at a higher resolution by genomic and other technologies to enable more precise targeting of subgroups of disease with new therapies" [48].

Collins and Varmus (2015) further elaborated that "The concept of precision medicine - prevention and treatment strategies that take individual variability into account - is not new; blood typing, for instance, has been used to guide blood transfusions for more than a century" [47]. The current study can serve the holistic concept of precision medicine and precision transplant medicine by incorporating NODAT's significant predictors in amalgamation with genomic data, for instance, HLA typing, to design an ideal management regimen for a successful transplant with the least possible postoperative complications. The current study's authors are aware of the limitation of not examining HLA profiling for transplant recipients due to institutional and ethical constraints. Nonetheless, it is critical for subsequent research to incorporate genetic and HLA profiling as potential risk factors and predictors of the evolution of new-onset diabetes after transplantation, the purpose of which should serve the concepts and applications of precision medicine and precision transplant medicine.

\section{Anticipated robust studies}

Future research requires incorporating larger samples and evaluating the heterogeneity among populations of interest. The results from our regression model (adjusted $R^{2}=0.464$, model accuracy $=46.4 \%$ ) indicated ambiguous predictors; therefore, researchers should test other potential risk factors, including HLA typing, ethnicity, socioeconomic backgrounds, polypharmacy, and coexisting medical or surgical comorbidities. Studies should aim for robust evidence by consulting non-Bayesian statistics and nAl models, and exploring other longitudinal study designs, controlled trials, systematic reviews, and meta-analytic studies while deploying reliable and replicable data analysis methods and statistical packages.

\section{Limitations of the study}

\section{Study design restrictions}

The current research is a longitudinal observational study of a retrospective design. According to the OCEBM, our study belongs to level-3 within the pyramidal hierarchy of the level of evidence [49]. Our research does have limitations other than those inherent to retrospective studies, including the sample size, which is relatively small; additionally, the number of cases (diabetic patients) and non-cases is relatively low. Other unique parameters for the sample cannot be fully known, for example, the HLA typing and the subethnicities of individuals who received the transplant. 
Besides, other demographic variables were unknown, including socioeconomic status and underlying pathologies affecting other body systems.

\section{Data analytics constraints}

Statistical analyses also possess limitations, such as the inflated type-1 statistical error due to multiple data analytics. In addition, the interpretation of causality that we implemented in our hypotheses may accept different viewpoints, including arguing the basis of Bradford Hill criteria when classifying specific variables into independent (predictors) and dependent (outcomes) [50]. Furthermore, all data analytics have some degree of error as per the aphorism of the renowned British statistician George Edward Box, "All models are wrong, but some are useful;" this applies to regression models and neural networks analysis [51]. The statistical packages also have implicit constraints; nevertheless, limitations related to statistical analyses or packages (software) can be overcome by integrating classical (frequentist) statistics and ML methods.

\section{Conclusions}

In reconciliation with frequentist statistics, our dual ML model validated several predictors that either negatively (protective factors) or positively (harmful factors) influenced the HbA1c levels and the possibility of developing NODAT, the majority of which were significant at $95 \% \mathrm{Cl}$. Hence, they influence the elective tendencies of specific individuals undergoing renal transplants to develop post-transplant diabetes. Transplant recipients who are HCV and CMV positive are predicted to develop NODAT. On the other hand, older recipients, with blood group O+ve, prior history of hemodialysis, a relatively high BMI before the transplant, and receiving higher doses of prednisolone following the transplant are also prone to have superior levels of HbAC1 following a successful transplant, which augment the probability of manifesting with posttransplant diabetes.

\section{Authors of Contribution}

AA conceptualized the study's aims, developed the research and statistical hypotheses, conducted data analytics, wrote the methods and results, developed the introduction and discussion sections, and coordinated the research team. AAA reviewed the literature, collected raw data, and wrote the introduction and discussion sections.

\section{References}

1. Davidson JA, Wilkinson A. New-onset diabetes after transplantation 2003 international consensus guidelines: An endocrinologist's view. Diabetes Care. 2004;27(3):805-12. http://doi.org/10.2337/diacare.27.3.805

PMid:14988309

2. Boyce EG, Lawson LA. Preprofessional curriculum in preparation for doctor of pharmacy educational programs. Am J Pharm Educ. 2009;73(8):155. http://doi.org/10.5688/aj7308155 PMid:20221348

3. YeX, Kuo HT, Sampaio MS, Jiang Y, Bunnapradist S. Risk factors for development of new $\square$ onset diabetes mellitus after transplant in adult lung transplant recipients. Clin Transplant. 2011;25(6):88591. http://doi.org/10.1111/j.1399-0012.2010.01383.x PMid:21175848

4. Montori VM, Basu A, Erwin PJ, Velosa JA, Gabriel SE, Kudva YC. Post-transplantation diabetes: A systematic review of the literature. Diabetes Care. 2002;25(3):583-92. http://doi. org/10.2337/diacare.25.3.583

PMid:11874952

5. Alberti KG, Zimmet PZ. Definition, diagnosis and classification of diabetes mellitus and its complications. Part 1: Diagnosis and classification of diabetes mellitus. Provisional report of a WHO consultation. Diabetic Med. 1998;15(7):539-53. http://doi.org/10.1002/ (SICI)1096-9136(199807)15:7<539:AID-DIA668>3.0.CO;2-S PMid:9686693

6. Araszkiewicz A, Bandurska-Stankiewicz E, Budzyński A Cypryk K, Czech A, Czupryniak L, et al. 2019 guidelines on the management of diabetic patients. A position of diabetes Poland. Clin Diabetol 2019;8(1):1-95.

7. Sharif A, Hecking M, de Vries AP, Porrini E, Hornum M, RasoulRockenschaub $\mathrm{S}$, et al. Proceedings from an international consensus meeting on posttransplantation diabetes mellitus: Recommendations and future directions. Am J Transplant. 2014;14(9):1992-2000. http://doi.org/10.1111/ajt.12850 PMid:25307034

8. Ponticelli C, Favi E, Ferraresso M. New-onset diabetes after kidney transplantation. Medicina. 2021;57(3):250. http://doi. org/10.3390/medicina57030250

PMid:33800138

9. Hjelmesæth J, Müller $F$, Jenssen $T$, Rollag H, Sagedal $S$, Hartmann $A$. Is there a link between cytomegalovirus infection and new-onset post-transplantation diabetes mellitus? Potential mechanisms of virus induced $\beta$-cell damage. Nephrol Dial Transplant. 2005;20(11):2311-5. http://doi.org/10.1093/ndt/ gfi033

PMid:16046502

10. Kuo HT, Sampaio MS, Ye X, Reddy P, Martin P, Bunnapradist S. Risk factors for new-onset diabetes mellitus in adult liver transplant recipients, an analysis of the Organ procurement and transplant network/united network for organ sharing database. Transplantation. 2010;89(9):1134-40. http://doi.org/10.1097/ TP.0b013e3181d2fec1

PMid:20386364

11. Fabiani $S$, Fallahi $P$, Ferrari $S M$, Miccoli $M$, Antonelli $A$ Hepatitis $C$ virus infection and development of Type 2 diabetes mellitus: Systematic review and meta-analysis of the literature. Rev Endocr Metab Disord. 2018;19(4):405-20. http://doi. org/10.1007/s11154-017-9440-1 PMid:29322398

12. Geer EB, Islam J, Buettner C. Mechanisms of glucocorticoidinduced insulin resistance: Focus on adipose tissue function 
and lipid metabolism. Endocrinol Metab Clin. 2014;43(1):75102. http://doi.org/10.1016/j.ecl.2013.10.005 PMid:24582093

13. Ullrich S, Berchtold S, Ranta F, Seebohm G, Henke G, Lupescu A, et al. Serum-and glucocorticoid-inducible kinase 1 (SGK1) mediates glucocorticoid-induced inhibition of insulin secretion. Diabetes. 2005;54(4):1090-9. http://doi.org/10.2337/ diabetes.54.4.1090

PMid:15793248

14. Øzbay LA, Smidt K, Mortensen DM, Carstens J, Jørgensen KA, Rungby J. Cyclosporin and tacrolimus impair insulin secretion and transcriptional regulation in INS-1E betacells. $\mathrm{Br} J$ Pharmacol. 2011;162(1):136-46. http://doi. org/10.1111/j.1476-5381.2010.01018.x

PMid:20825407

15. Kahn SE. The relative contributions of insulin resistance and beta-cell dysfunction to the pathophysiology of Type 2 diabetes. Diabetologia. 2003;46(1):3-19. http://doi.org/10.1007/ s00125-002-1009-0

PMid: 12637977

16. Vincenti F, Friman S, Scheuermann E, Rostaing L, Jenssen T, Campistol JM, et al. Results of an international, randomized trial comparing glucose metabolism disorders and outcome with cyclosporine versus tacrolimus. Am JTransplant. 2007;7(6):150614. http://doi.org/10.1111/j.1600-6143.2007.01749.x PMid:17359512

17. Torres A, Hernández D, Moreso F, Serón D, Burgos MD, Pallardó LM, et al. Randomized controlled trial assessing the impact of tacrolimus versus cyclosporine on the incidence of posttransplant diabetes mellitus. Kidney Int Rep. 2018;3(6):130415. http://doi.org/10.1016/j.ekir.2018.07.009 PMid:30450457

18. Yu H, Kim H, Baek $\mathrm{CH}$, Baek SD, Jeung S, Han DJ, et al. Risk factors for new-onset diabetes mellitus after living donor kidney transplantation in Korea-a retrospective single center study. BMC Nephrol. 2016;17(1):106. http://doi.org/10.1186/ s12882-016-0321-8

PMid:27473469

19. Al-Imam A, Ali MA, Al-Mukhtar SE. The spectrum of biopsyproved kidney disease: A retrospective single center study in Erbil-Iraq. Asian J Med Sci. 2019;10(2):46-51.

20. Woodward RS, Schnitzler MA, Baty J, Lowell JA, LopezRocafort L, Haider $\mathrm{S}$, et al. Incidence and cost of new onset diabetes mellitus among US wait-listed and transplanted renal allograft recipients. Am J Transplant. 2003;3(5):590-8. http://doi. org/10.1034/j.1600-6143.2003.00082.x

PMid: 12752315

21. Palepu S, Prasad GR. New-onset diabetes mellitus after kidney transplantation: Current status and future directions. World J Diabetes. 2015;6(3):445-55. http://doi.org/10.4239/wjd. v6.i3.445

\section{PMid:25897355}

22. Cosio FG, Pesavento TE, Osei K, Henry ML, Ferguson RM. Post-transplant diabetes mellitus: increasing incidence in renal allograft recipients transplanted in recent years. Kidney Int. 2001;59(2):732-7. http://doi. org/10.1046/j.1523-1755.2001.059002732.x

PMid:11168956

23. Bonato V, Barni R, Cataldo D, Collini A, Ruggieri G, De Bartolomeis $\mathrm{C}$, et al. Analysis of posttransplant diabetes mellitus prevalence in a population of kidney transplant recipients. Transplant Proc. 2008;40(6):1888-90. http://doi.org/10.1016/j. transproceed.2008.05.045

PMid: 18675080

24. Shah T, Kasravi A, Huang E, Hayashi R, Young B, Cho YW, et al.
Risk factors for development of new-onset diabetes mellitus after kidney transplantation. Transplantation. 2006;82(12):1673-6. PMid:17198258

25. Cole EH, Prasad GR, Cardella CJ, Kim JS, Tinckam KJ, Cattran DC, et al. A pilot study of reduced dose cyclosporine and corticosteroids to reduce new onset diabetes mellitus and acute rejection in kidney transplant recipients. Transplantat Res. 2013;2(1):1-7. http://doi.org/10.1186/2047-1440-2-1 PMid:23369458

26. Zolota A, Miserlis G, Solonaki F, Tranda A, Antoniadis N, Imvrios $G$, et al. New-onset diabetes after transplantation: Comparison between a cyclosporine-based and a Tacrolimus-based immunosuppressive regimen. Transplant Proc. 2018;50(10):3386-91. http://doi.org/10.1016/j. transproceed.2018.08.037

PMid:30577210

27. Hjelmesaeth J, Sagedal S, Hartmann A, Rollag H, Egeland T, Hagen $\mathrm{M}$, et al. Asymptomatic cytomegalovirus infection is associated with increased risk of new-onset diabetes mellitus and impaired insulin release after renal transplantation. Diabetologia. 2004;47(9):1550-6. http://doi.org/10.1007/ s00125-004-1499-z

PMid: 15338129

28. Sinangil A, Celik V, Barlas S, Koc Y, Basturk T, Sakaci T, et al. The incidence of new onset diabetes after transplantation and related factors: Single center experience. Nefrologia. 2017;37(2):181-8. http://doi.org/10.1016/j.nefro.2016.11.022 PMid:28262264

29. Abbott KC, Lentine KL, Bucci JR, Agodoa LY, Koff JM, Holtzmuller KC, et al. Impact of diabetes and hepatitis after kidney transplantation on patients who are affected by hepatitis C virus. J Am Soc Nephrol. 2004;15(12):3166-74. http://doi. org/10.1097/01.ASN.0000145439.48387.BF PMid: 15579520

30. Baid S, Tolkoff-Rubin N, Farrell ML, Delmonico FL, Williams WW. Tacrolimus-associated posttransplant diabetes mellitus in renal transplant recipients: Role of hepatitis $\mathrm{C}$ infection. Transplant Proc. 2002;34(5):1771-3. http://doi.org/10.1016/ s0041-1345(02)03060-9

PMid:12176569

31. Cruzado JM, Bestard O, Grinyó JM. Impact of extrahepatic complications (diabetes and glomerulonephritis) associated with hepatitis $C$ virus infection after renal transplantation. Hepatitis $C$ in renal disease, hemodialysis and transplantation. Contrib Nephrol. 2012;176:108-16. http://doi.org/10.1159/000332389 PMid:22310786

32. Shin JI, Palta M, Djamali A, Astor BC. Higher pre-transplantation hemoglobin $\mathrm{A} 1 \mathrm{c}$ is associated with greater risk of posttransplant diabetes mellitus. Kidney Int Rep. 2017;2(6):1076-87. PMid:29270516

33. Tillmann FP, Schmitz M, Rump LC, Quack I. Impact of low-dose steroids on HbA1c levels and development of pre-diabetes and NODAT in non-diabetic renal transplant recipients on longterm follow-up. Int Urol Nephrol. 2018;50(4):771-7. http://doi. org/10.1007/s11255-017-1754-0 PMid:29197933

34. Boots JM, Van Duijnhoven EM, Christiaans MH, WolffenbuttelBH, van Hooff JP. Glucose metabolism in renal transplant recipients on tacrolimus: The effect of steroid withdrawal and tacrolimus trough level reduction. J Am Soc Nephrol. 2002;13(1):221-7. http://doi.org/10.1681/ASN.V131221 PMid: 11752041

35. Al-Imam A, Abdul-Wahaab IT, Konuri VK, Sahai A, Al-Shalchy AK. Unification of frequentist inference and machine learning for pterygomaxillary morphometrics. Folia Morphol. 
2021;80(3):625-41. http://doi.org/10.5603/FM.a2020.0149 PMid:33438189

36. Al-Imam A, Motyka MA, Jędrzejko MZ. Conflicting opinions in connection with digital superintelligence. IAES Int J Artif Intell. 2020;9(2):336-48. http://doi.org/10.11591/ijai.v9.i2.pp336-348

37. Al-Imam A, Motyka MA, Sahai A, Konuri VK. The "march of progress": From cosmic singularity to digital singularity. Curr Trends Inf Technol. 2020;10(1):1-8.

38. Al-Imam A. Morphometry of Pterygomaxillary Landmarks in Reference to Gender and Laterality [Dissertation on the Internet] Iraq: University of Baghdad; 2021. http://doi.org/10.13140/ RG.2.2.28077.00480

39. Al-Imam A, Khalid U, Al-Doori HJ. Clustering analysis of coronavirus disease 2019 pandemic. Asian J Med Sci. 2020;12(2):108-13.

40. Motyka MA, Al-Imam A, Aljarshawi MH. SARS-CoV-2 pandemic as an anomie. Przestrzeń Społeczna (Social Space). 2020;20:111-44.

41. Al-Imam A, Khalid U, Al-Doori HJ. Predictive epidemiology for SARS-CoV-2 pandemic in Iraq. Asian J Med Sci. 2021;12(3):121-4. https://doi.org/10.3126/ajms.v12i3.33402

42. Worldometer Real Time World Statistics. Worldometer; 2020. Available from: https://www.worldometers.info [Last accessed on 2021 Sep 28].

43. Siddiqi HK, Libby P, Ridker PM. COVID-19 a vascular disease. Trends Cardiovasc Med. 2021;31(1):1-5. http://doi. org/10.1016/j.tcm.2020.10.005

PMid:33068723
44. Roberts KA, Colley L, Agbaedeng TA, Ellison-Hughes GM, Ross MD. Vascular manifestations of COVID-19thromboembolism and microvascular dysfunction. Front Cardiovasc Med. 2020;7:215. http://doi.org/10.3389/ fcvm.2020.598400

PMid:33195487

45. Al-Imam A, Motyka MA, Al-Doori HJ. Surface web merits for SARS-CoV-2 pandemic in Iraq. J Facult Med Baghdad. 2020;62(4):117-27.

46. Al-Doori HJ, Alwan SH, Al-Imam A, Hussein HA, Al-Shalchy AK. SARS-CoV-2 and pediatric encephalopathy: Google analytics and predictive modeling. Asian J Med Sci. 2021;12(11):29-34.

47. Collins FS, Varmus $H$. A new initiative on precision medicine. N Engl J Med. 2015;372(9):793-5. http://doi.org/10.1056/ NEJMp1500523

PMid:25635347

48. Ashley EA. Towards precision medicine. Nat Rev Genet. 2016;17(9):507-22. http://doi.org/10.1038/nrg.2016.86 PMid:27528417

49. University of Oxford. The Centre for Evidence-Based Medicine. Available from: https://www.cebm.net [Last accessed on 2021 Sep 09].

50. Al-Imam A, Gorial FI, Al-shalchy A. A novel unusual manifestation of $\mathrm{CH}$-Alpha as acute metabolic disturbances: Case report and big data analytics. J Facult Med Baghdad. 2020;62(1-2):41-7.

51. Al-Imam A, Sahai A, Al-Derzi AR, Al-Shalchy A, Abdullah F. All models are wrong, but some are useful: On the non-bayesian statistical robustness of Hilton's law. Eur J Anat. 2020;24(1):75-8. 